\title{
Mechanically Induced Reactive Gliosis Causes ATP-Mediated Alterations in Astrocyte Stiffness
}

\author{
William J. Miller, ${ }^{1}$ Ilya Leventhal, ${ }^{1}$ David Scarsella, ${ }^{1}$ Philip G. Haydon, ${ }^{5}$ \\ Paul Janmey, ${ }^{1,3,4}$ and David F. Meaney ${ }^{1,2}$
}

\begin{abstract}
Reactive gliosis is a process triggered in astrocytes after traumatic injury, yet the exact consequences of gliosis on cellular survival and neural regenerative processes in the injured brain remain only partly understood. One recently discovered feature influencing neuronal growth and differentiation is the physical stiffness of the environment surrounding pioneering neurites. In this study, the mechanical properties of cultured cortical astrocytes are measured following a mechanical stretch injury that induces reactive gliosis. In mechanically injured cultures, there was a significant increase in glial fibrillary acidic protein (GFAP) immunoreactivity $24 \mathrm{~h}$ following a rapid, transient $15 \%$ strain. In these same cultures, astrocytes in the surrounding region-the "mechanical penumbra"-also exhibited increased GFAP immunoreactivity compared to naive cultures. Correlated with these changes in GFAP was a general softening of the non-nuclear regions of the astrocytes, both in the injured and penumbra cells, as measured by atomic force microscopy (AFM). The elastic modulus in naive cultures was observed to be $57.7 \pm 5.8 \mathrm{kPa}$ in non-nuclear regions of naive cultures, while $24 \mathrm{~h}$ after injury the modulus was observed to be $26.4 \pm 4.9 \mathrm{kPa}$ in the same region of injured cells. In the penumbra of injured cultures, the modulus was $23.7 \pm 3.6 \mathrm{kPa}$. Alterations in astrocyte stiffness in the area of injury and mechanical penumbra were ameliorated by pretreating cultures with a nonselective P2 receptor antagonist (PPADS). Since neuronal cells generally prefer softer substrates for growth and neurite extension, these findings may indicate that the mechanical characteristics of reactive astrocytes are favorable for neuronal recovery after traumatic brain injury.
\end{abstract}

Key words: glia cell response to injury; in vitro studies; traumatic brain injury

\section{Introduction}

$\mathbf{P}$ AST WORK SHOWS ASTROCYTES PERFORM many important functions within the central nervous system (CNS), including the release of neurotransmitters, the secretion of trophic factors, and the synthesis and release of molecules to shape the extracellular matrix (Sofroniew, 2005). With the close proximity of astrocytic end feet to the chemical synapse of some neurons (Ventura and Harris, 1999) and the connectivity of a single astrocyte to several hundred neighboring dendrites (Halassa et al., 2007), it is not surprising that recent reports show that astrocytes can shape the process of synaptic neurotransmission (Araque et al., 1998a,b; Kang et al., 1998; Fiacco and McCarthy, 2004). Perhaps equally important is the active role that the astrocytes play in influencing the fate of neurons during the course of disease or following damage in the CNS (Halassa et al., 2007). Currently, though, there is an incomplete view on how the changes in astrocyte behavior-including the functional, structural, and molecular alterations-following traumatic brain injury (TBI) will contribute to the repair process after injury.

One of the most dramatic changes in astrocytes following focal TBI is the reactive gliosis surrounding the lesion. In general, gliosis is a process that involves proliferation, increased process length, production of extracellular matrix and upregulation of glial fibrillary acidic protein (GFAP) in astrocytes (Pekny and Nilsson, 2005). Despite the growing number of reports on how astrocytes can control neuronal fate

Departments of ${ }^{1}$ Bioengineering, ${ }^{2}$ Neurosurgery, ${ }^{3}$ Physiology, and the ${ }^{4}$ Institute of Medicine and Engineering, University of Pennsylvania, Philadelphia, Pennsylvania.

${ }^{5}$ Department of Neuroscience, Tufts University, Boston, Massachusetts. 
and regeneration after injury, there is one surprisingly simple physical property of reactive astrocytes related to the change in its cytoskeleton (i.e., the intrinsic mechanical properties or, more generally, stiffness of the cell) which has been largely overlooked. In general, substrate stiffness is increasingly known for its importance in cell attachment, motility, and process extension, especially in neuronal cells (Pelham and Wang, 1997; Lo et al., 2000; Balgude et al., 2001; Wang et al., 2001; Flanagan et al., 2002). Unlike astrocytes, which grow best on harder substrates (Georges et al., 2006), neurons prefer soft substrates, with neurite branching decreasing significantly when substrate stiffness is greater than that measured in human gray matter (Pelham and Wang, 1997; Lo et al., 2000; Balgude et al., 2001; Flanagan et al., 2002; Discher et al., 2005; Lu et al., 2006). Indeed, astrocyte monolayers in vitro provide a more favorable environment for neurite outgrowth and neuronal attachment (Powell et al., 1997) when compare to astrocyte conditioned media, but this finding remains largely unexplained. Given the cytoskeletal alterations that occur within reactive astrocytes after mechanical injury, a natural question arises: Will reactive astrocytes show a change in their mechanical properties, and what mechanism mediates this alteration in stiffness?

In this study, we tested if cultured astrocytes show changes in their cytoskeletal structure and mechanical stiffness following traumatic mechanical injury. We used an in vitro model of traumatic mechanical injury to establish conditions that would lead to astrocytic reactivity $24 \mathrm{~h}$ following injury, and then used atomic force microscopy (AFM) to compare the elastic properties of individual reactive astrocytes to control, uninjured astrocytes. In addition, we determined whether changes in cellular stiffness and immunoreactivity extend beyond the initial area of mechanical injury in vitro, and if there is a pharmacological means to attenuate stretch-induced changes in stiffness across astrocyte monolayers. We found significant alterations appeared in the stiffness of mechanically injured astrocytes and that these changes were not restricted to the region of initial mechanical injury. In addition, we determined stretch-induced changes were blocked using an inhibitor of a specific subtype of purinergic receptors prior to injury. Together, these data show that the mechanical properties of astrocytes is a new factor to consider when interpreting the role of reactive gliosis following mechanical trauma, and there may be readily available therapeutic targets which influence this feature of reactive astrocytes after TBI.

\section{Methods}

\section{Astrocyte cell culture and drug treatment}

Pure cortical astrocyte cultures were generated from E18 Sprague-Dawley rat embryos (Charles River Laboratories, Wilmington, MA) according to animal welfare guidelines and using procedures approved by the University of Pennsylvania's Institutional Animal Care and Use Committee (IACUC). Briefly, brains were dissected from the embryos, and the meninges were removed. The cortices were dissected and dissociated by incubating in Neurobasal media (Invitrogen Corp., Carlsbad, CA) with trypsin $(0.3 \mathrm{mg} / \mathrm{ml}$; SigmaAldrich, St. Louis, MO) + DNase I $(0.2 \mathrm{mg} / \mathrm{ml}$; Amersham Biosciences, Piscataway, NJ) at $37^{\circ} \mathrm{C}, 5 \% \mathrm{CO}_{2}$. Enzymatic activity was inhibited after $20 \mathrm{~min}$ by adding soybean trypsin inhibitor $(0.5 \mathrm{mg} / \mathrm{ml}$; Gibco, Grand Island, NY). The tissue was mechanically disrupted by pipetting, then centrifuged for $5 \mathrm{~min}$ at $1000 \mathrm{rpm}$ and resuspended in Dulbecco's Modified Eagle's Medium (DMEM; Cambrex Bio Science, Walkersville, Inc., Walkersville, MD) $+5 \%$ fetal bovine serum (FBS; Hyclone, Logan, UT). Cells were filtered sequentially through a $60-\mu \mathrm{m}$ and $28-\mu \mathrm{m}$ Nitex Mesh (Cross Wire Cloth \& Manufacturing Co., Bellmawr, NJ) and plated onto poly-L-lysine (PLL; Sigma)-treated T75 tissue culture flasks (Fischer Scientific, Inc., Pittsburgh, PA) at a concentration of $1 \times 10^{5}$ cells $/ \mathrm{ml}$. Medium was changed every 3-4 days.

At 13 days in vitro (DIV), cells were placed on an orbital shaker and shaken at $250 \mathrm{rpm}$ overnight at $37^{\circ} \mathrm{C}, 5 \% \mathrm{CO}_{2}$ to remove loosely adherent cells that included neurons and microglia. Flasks were rinsed with saline solution before adding $4 \mathrm{ml}$ of trypsin/EDTA ( $0.25 \%$; Invitrogen) for 2-3 min at $37^{\circ} \mathrm{C}$, and then mechanically disrupted to dislodge the cell layer from the flask surface. DMEM $+5 \%$ FBS was added to inhibit enzymatic activity. The cells were centrifuged for $5 \mathrm{~min}$ at $1000 \mathrm{rpm}$ and resuspended in DMEM $+5 \%$ FBS. The cell suspension was diluted to $1 \times 10^{5}$ cells $/ \mathrm{ml}$ and plated onto PLL-treated silicone-based elastic membranes (cured Sylgard 186/Sylgard 184 at a 7:4 mix; Dow Corning, Midland, MI). Medium was changed at $24 \mathrm{~h}$ and then every 3-4 days until use after 13-14 DIV, at which point cultures had reached confluency. Cultures were determined to be $>95 \%$ pure astrocytes by immunochemistry for GFAP (astrocytes), type-3 beta-tubulin (neurons), and CNPase (oligodendrocytes) counterstained with Hoechst. Cultures were discarded if not confluent.

Prior to mechanical injury, the medium was replaced with DMEM $+5 \%$ FBS. This medium remained on cultures for the entire duration of the experiment. For a selected group of experiments, cultures were injured in the presence of pyridoxalphosphate-6-azophenyl-2',4'-disulphonic acid (PPADS; $10 \mu \mathrm{M}$; Sigma), a nonselective antagonist of P2 purinergic receptors. For another group of experiments, cultures were pretreated with the extracellular ATP degrading enzyme apyrase $(20 \mathrm{U} / \mathrm{mL}$; Sigma). Inhibitors were brought to their final concentration in the media formulation above, and cultures were incubated with inhibitors beginning $15 \mathrm{~min}$ prior to mechanical injury and continuing for the complete 24-h period following injury.

\section{Mechanical injury to astrocyte monolayers}

We used a system developed in past studies to stretch a portion of the astrocyte monolayer, controlling both the magnitude and duration of the stretch insult. A rapid, transient air pressure pulse was applied to the culture surface, deflecting the membrane downward. A metal plate beneath the culture restricted the deflection of the elastic membrane to a $2 \mathrm{~mm} \times 18 \mathrm{~mm}$ rectangular region, approximately $12 \%$ of the entire membrane surface (Fig. 1). Marks on the membrane readily distinguished the region of direct stretch from the adjacent area of membrane that did not deform (Lusardi et al., 2004). The geometry of the stretched area created a nearly uniform uniaxial strain field throughout the stretched region (Lusardi et al., 2004), where the maximum membrane strain is in proportion to the applied pressure. The time to achieve peak strain $(20 \mathrm{~ms})$ and magnitude of the strain $(5 \%, 15 \%$, 
A
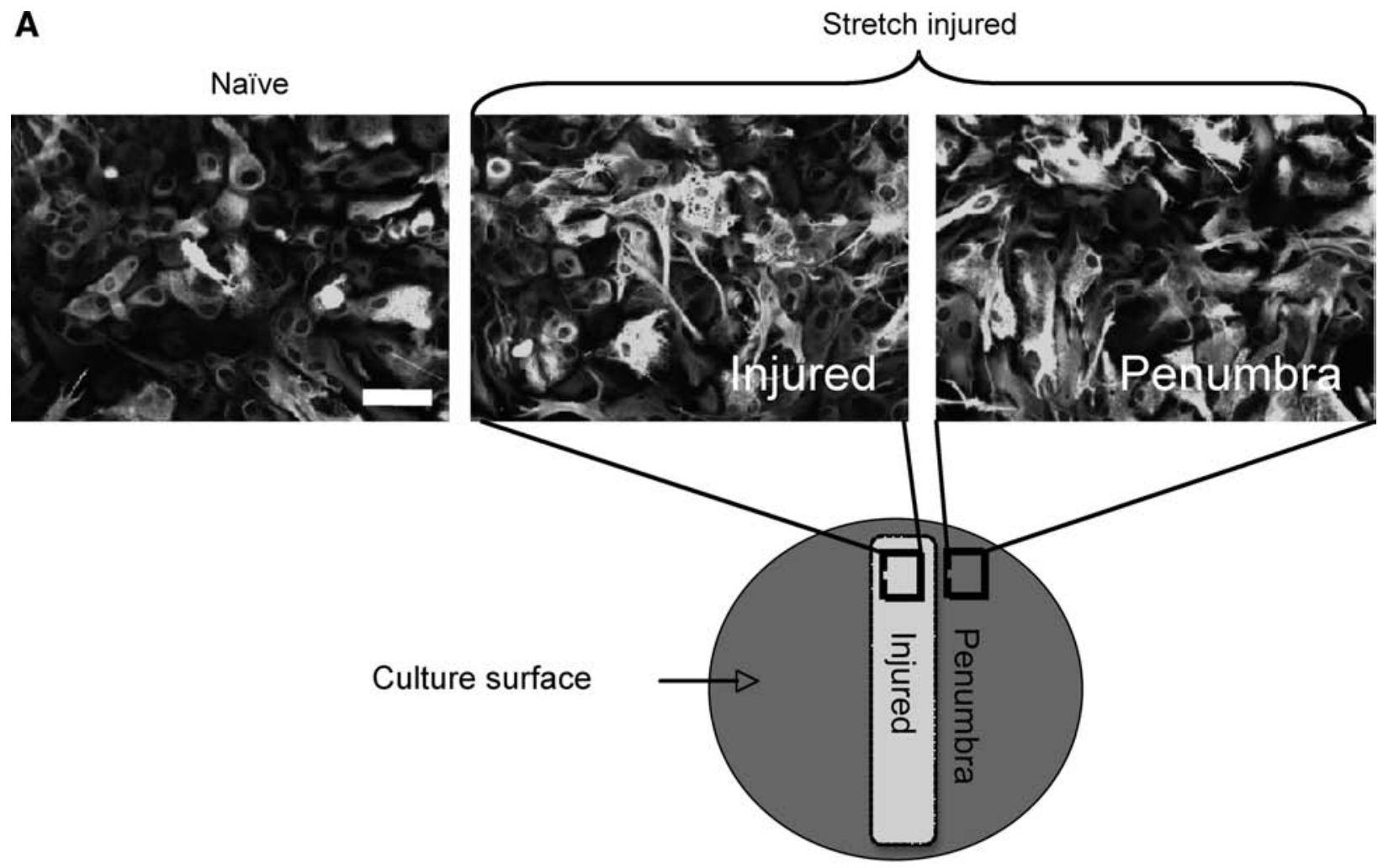

B

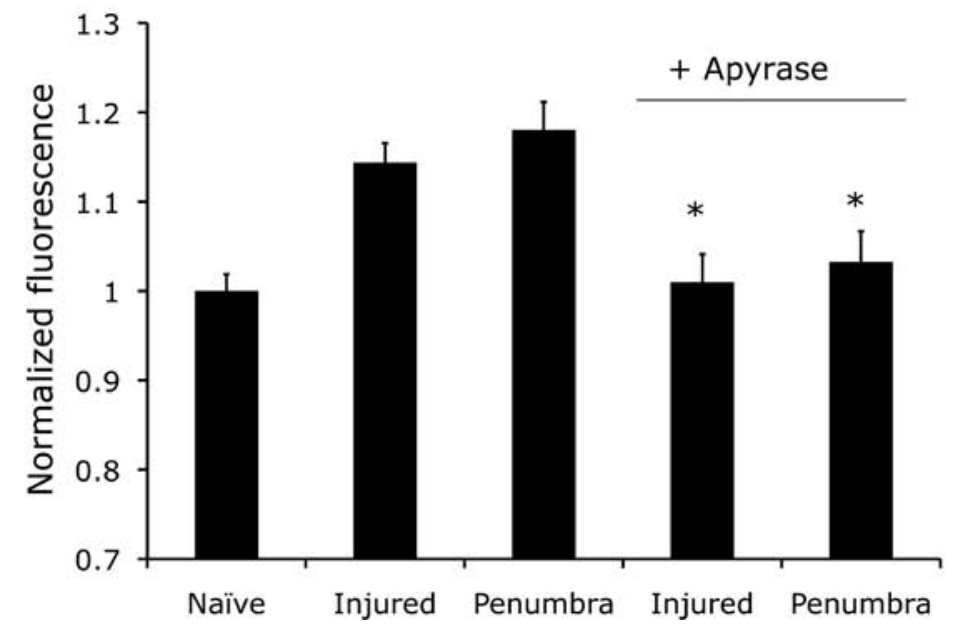

FIG. 1. Mechanical injury to cultured astrocytes produces reactive gliosis within and distant from the site of mechanical injury. (A) Overview of the in vitro system used to mechanically injure primary cortical astrocytes. The system allows one to stretch only a subregion of the entire culture. Immunocytochemical analysis with anti-glial fibrillary acidic protein (GFAP) antibody showed an increase in GFAP immunoreactivity $24 \mathrm{~h}$ following stretch. (B) Quantitative analysis of immunofluorescence intensity when compared to naive samples from the same culture region showed a significant increase in GFAP immunoreactivity (asterisk; $p<0.05$ ) across the entire culture. The stretch-induced increase in immunoreactivity was eliminated by treating the cultures with apyrase $(20 \mathrm{U} / \mathrm{ml})$ to enzymatically degrade extracellular ATP. Scale bar $=50 \mu \mathrm{m}$.

25\%) were controlled to simulate mild TBI (Shreiber et al., 1997). We did not observe any overt changes in the astrocyte morphology immediately following stretch, and all cells were adhered to the membrane following injury. Additionally, we did not detect any changes in viability one day following injury using propidium iodide staining. We termed the area of the culture where the membrane deformed as the "injured" region, while the region surrounding the region of stretchinjured cells was defined as the "mechanical penumbra" (i.e., the area that experienced no direct mechanical deformation) (Lusardi et al., 2004). All cultures were stretched once and returned to the incubator environment for $24 \mathrm{~h}$ before conducting AFM measurements or GFAP immunocytochemistry. 


\section{Evaluating GFAP immunoreactivity in cultured astrocytes}

At $24 \mathrm{~h}$ following stretch injury, cultures were rinsed with PBS and fixed in $4 \%$ paraformaldehyde for $15 \mathrm{~min}$. Cells were permeabilized in $0.2 \%$ Triton (Sigma) in Tris-buffered saline (TBS) for $5 \mathrm{~min}$. Primary monoclonal GFAP antibody (1:1000; Chemicon, Temecula, CA) in 5\% normal goat serum (NGS) was added, and the cultures were incubated overnight at $4^{\circ} \mathrm{C}$. The cells were rinsed $2 \times$ with TBS and incubated for $30 \mathrm{~min}$ in $5 \%$ NGS and then the secondary antibody (Alexa Fluor 488, 1:1000; Chemicon) was added for $1 \mathrm{~h}$. The cells were rinsed and stored at $4{ }^{\circ} \mathrm{C}$ until imaging. For all experiments, no primary and no secondary antibody controls revealed no nonspecific labeling.

Immunoreactivity was quantified by using Metamorph software (Universal Imaging, West Chester, PA) to compute an average fluorescence for a full-field image $(350 \mu \mathrm{m} \times$ $350 \mu \mathrm{m})$. A threshold was set for the images to include only cellular areas, and average immunofluorescence was recorded. In stretched cultures, three to six images were taken randomly from the mechanically injured region, and four to 10 images were taken from the adjacent, uninjured (penumbra) regions. Image thresholds and image acquisition settings were kept constant throughout each analysis step. In unstretched cultures, six to 10 images were taken randomly throughout the culture. Immunoreactivity was compared from week to week by including at least three naive controls each week and normalizing each week's immunoreactivity data to the naive controls for that week.

\section{Atomic force microscopy measurement of astrocytes mechanical properties}

Images were acquired using a Bioscope AFM with a Nanoscope IIIa controller (Veeco Instruments) and a DAFM-2X Dimension Head Scanner (Veeco). Images were obtained in contact mode using silicon nitride DNP cantilevers (Veeco), which are nominally $200 \mu \mathrm{m}$ long, with $20-\mu \mathrm{m}$-wide legs. Contact mode images were taken in DMEM media at room temperature with scan rates of $30-100 \mu \mathrm{m} / \mathrm{s}$ and scan sizes of $10-100 \mu \mathrm{m}$ in each direction. These images were used to identify nuclear and non-nuclear regions of each astrocyte. After imaging a region of the culture, force curves were collected by indenting the cantilever onto the cell surface while holding the $x, y$-axis constant. The nominal spring constant of these cantilevers is reported to be $0.06 \mathrm{~N} / \mathrm{m}$, although actual spring constants were determined using resonance frequency measurements of the tip prior to each experiment. The sensitivity of each cantilever was calibrated before use by lowering the cantilever onto a glass slide. After the cantilever reached the surface of the cell, it was lowered at least $300 \mathrm{~nm}$ towards the cell surface at a rate of $1 \mathrm{~Hz}$.

The apparent Young's modulus, E, was determined by fitting deflection curves for each culture using Sneddon's modification of the Hertzian model (Sneddon, 1965; Landau and Lifshitz, 1970):

$$
\mathrm{F}=\frac{4 \mathrm{ER}^{12} \delta^{32}}{3\left(1-\nu^{2}\right)}
$$

where $\mathrm{R}$ is the radius curvature at the apex, $\mathrm{F}$ is the loading force, $\mathrm{E}$ is the elastic Young's modulus, $\nu$ is the Poisson ratio
( $\nu=0.5$; based on Sato et al. [1990]), and $\delta$ was the deflection of the cantilever (Sneddon, 1965).

\section{Statistical analysis}

Data are reported as mean \pm standard error except where specified otherwise. Significance for GFAP immunoreactivity and for differences in Young's modulus was determined by analysis of variance (ANOVA) with strain (injured vs. naive) and culture location (stretched vs. penumbra) as groups. Curve fitting for the AFM experiments was determined by linear regression and by a chi-squared test for goodness of fit. ANOVA testing followed by Tukey posthoc analysis was used to detect significant differences between untreated and PPADS-treated cultures.

\section{Results}

\section{Mechanical stretch induces an ATP-dependent increase in GFAP immunoreactivity 24 hours following injury}

Our first objective was to establish the mechanical conditions leading to a marked increase in GFAP immunoreactivity $24 \mathrm{~h}$ following stretch injury. We used a range of peak membrane strains that did not cause any significant cell death, indicated by the absence of propidium iodide staining $24 \mathrm{~h}$ following injury (data not shown). Through a series of preliminary studies at three peak strain values $(5 \%, 15 \%$, and $25 \%$ ), we found that, although there was an increase in immunoreactivity following 5\% and 15\% stretch, the threshold for a significant increase in GFAP immunoreactivity occurred following a $15 \%$ strain injury (Fig. 1A,B). At the $15 \%$ level of mechanical injury, astrocytes showed no increased cell density one day after injury, and showed only nominal increases in process length (Fig. 1A). Astrocytes in the penumbra regions of the injury, defined as the regions immediately adjacent to the stretch (Fig. 1A), also showed similar morphological changes and stained significantly more intensely for GFAP following 15\% stretch injury when compared to naive cultures $(p<0.05$, Fig. 1B). There was no difference in GFAP immunoreactivity between stretched and penumbra astrocytes. Based on past studies showing exogenous ATP is capable of initiating reactive gliosis in astrocytes (Abbracchio et al., 1996; Brambilla et al., 2002; Neary et al., 2005), we pretreated cultures with apyrase to enzymatically degrade extracellular ATP in the media for $24 \mathrm{~h}$ following injury. Apyrase treatment consistently reduced the stretch-induced GFAP immunoreactivity to control levels in both the injured and penumbra regions (Fig. 1B), showing that extracellular ATP was a key factor mediating stretch-induced gliosis.

\section{Mechanical properties of cultured astrocytes vary regionally}

We next completed measurements of mechanical properties in naive, unstretched cultures to detect if any regional variations in mechanical properties occurred prior to acquiring properties in stretch-injured cultures. Recent reports show the significant difference between overall measurements of cell stiffness and isolated cell nucleus, with the nucleus showing a more strongly nonlinear behavior and stiffer response when compared to bulk properties of individual cells 

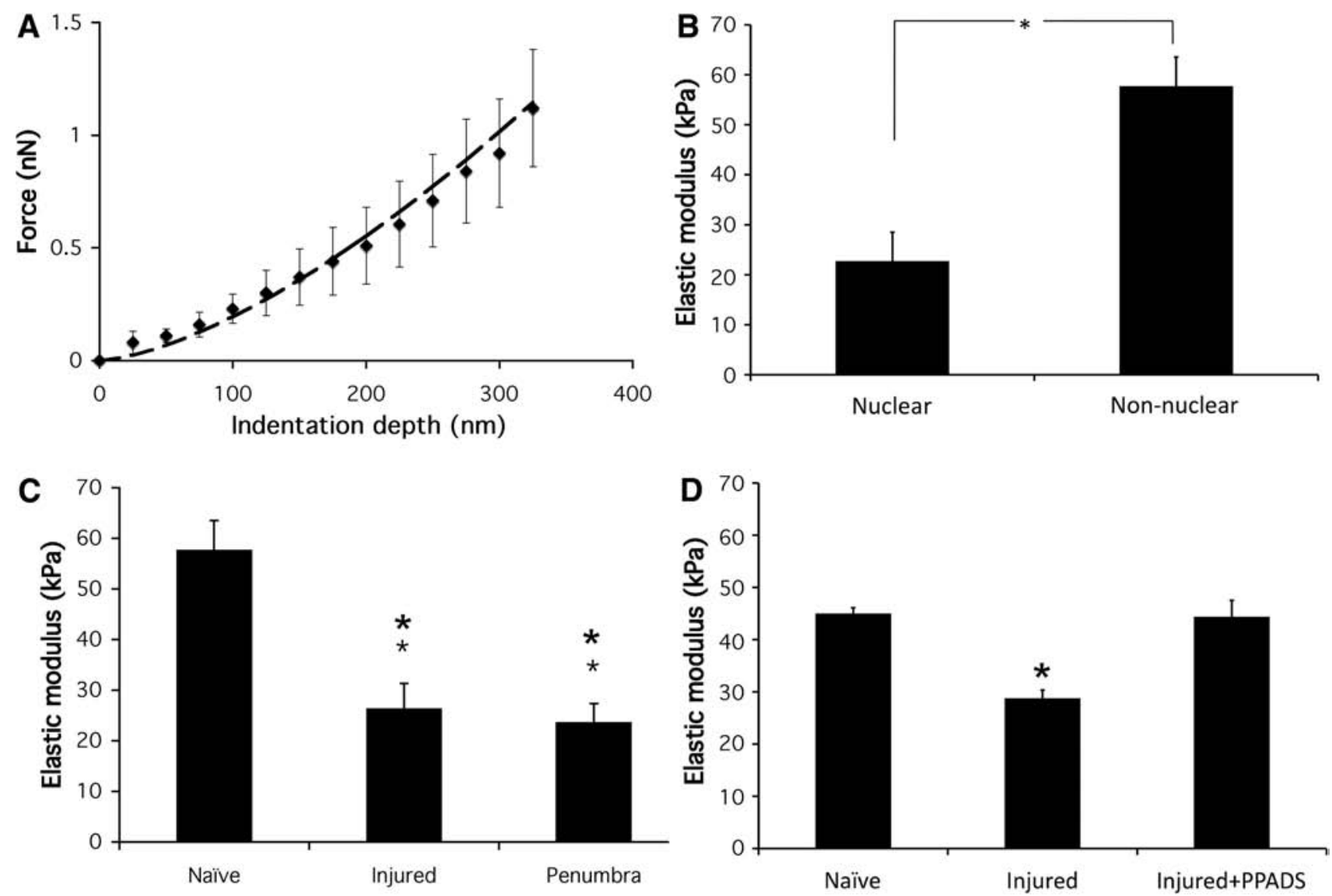

FIG. 2. Alterations in mechanical properties of reactive astrocytes are mediated by the P2 subtype purinergic receptors. (A) Atomic force microscopy was used to indent the surface of cultured astrocytes and measure the elastic modulus for individual cells. Shown is the average force-deflection curve (filled symbols; mean + SD) obtained for naïve astrocytes indented in non-nuclear regions. Force deflection curves were fit (dashed line) to determine the elastic modulus. (B) Indentation data revealed an estimated elastic modulus in naive cultures that was much stiffer over non-nuclear regions in comparison to nuclear areas. (C) In mechanically injured cultures, the astrocytes showed a significant softening (asterisk; $p<0.05)$ in both the injured area and the mechanical penumbra, similar to the changes in glial fibrillary acidic protein (GFAP) immunoreactivity observed in both regions. No difference in the elastic modulus was detected between the injured and penumbra regions. (D) The mechanically induced softening was prevented by pretreating cultures with a nonselective P2 receptor antagonist (PPADS, $10 \mu \mathrm{M}$ ).

(Caille et al., 2002; Dahl et al., 2005). We measured only the elastic, rather than viscoelastic, properties of individual astrocytes based on the role of elastic substrate stiffness properties on neuronal morphology and synaptic development. We made separate measurements for nuclear and non-nuclear regions of the cells, using the image acquired from the contact mode on the AFM to develop a three-dimensional surface profile of each cell to discriminate between nuclear regions, which showed a much higher height relative to the substrate, from non-nuclear regions which showed a flat profile relative to the membrane culture surface. In naive cultures, there was a significant increase in the elastic modulus measured in nonnuclear regions of individual astrocytes $(57.7 \pm 5.8 \mathrm{kPa}$; $n=27$ cells) when compared to properties measured in the nuclear region $(22.7 \pm 5.8 \mathrm{kPa})$. Given that the indentations above the nuclear region were likely a combination of elastic properties from both the cytoskeleton and cell nucleus, we concentrated the remainder of our analysis only on measurements from the non-nuclear region, which we considered a more consistent reflection of only the cytoskeletal alterations caused by stretch injury.

\section{Mechanically injured astrocytes show regional changes in cell stiffness}

We next tested if the changes in GFAP immunoreactivity also corresponded to a change in the mechanical properties of individual cells. Measurements at 1 day following mechanical injury revealed a significant reduction, or softening, of the elastic modulus in astrocytes from the injured region $(26.4 \pm 4.9 \mathrm{kPa}$ [injured; $n=18$ cells] vs. $57.7 \pm 5.83 \mathrm{kPa}$ [naive; $n=27] ; p<0.05$; Fig. 2C). The softening effect also appeared in astrocytes from the mechanical penumbra region, with a significant reduction in comparison to naive controls $(23.7 \pm 3.6 \mathrm{kPa}$ compared to $57.7 \pm 5.8 \mathrm{kPa}$ in naive cultures; $p<0.05$; Fig. 2C). Interestingly, we detected no significant difference in the measured elastic modulus between the injured and penumbra regions of the culture, similar to the 
observations on GFAP immunoreactivity between these two areas of the culture.

\section{Changes in cell stiffness following mechanical injury are inhibited by blocking $P 2$ type purinergic receptors}

With evidence showing mechanical stretch injury of astrocytes produces significant changes in the cell stiffness in both the injured and penumbra region, we next considered if there was a pharmacological means to ameliorate this effect. Concentrating our efforts on ATP-mediated mechanisms was a natural direction to pursue, given the role of extracellular ATP in mediating the reactive gliosis across both the injured and penumbra regions of the culture (Fig. 1). Rather than broadly testing all ATP-mediated receptors and mechanisms, though, we focused on inhibiting the P2 subtype of purinergic receptors because of the past demonstrated role for these receptors in both ERK phosphorylation and AkT activation, considered two possible steps leading to reactive gliosis (Munsch et al., 1998; Neary et al., 1998, 1999, 2003; Lenz et al., 2000). We measured changes in the mechanical properties of individual cells across the entire culture surface, maintaining a balance between cells in the injured and mechanical penumbra region. Pretreating cultures with a nonselective P2 receptor antagonist (PPADS; $10 \mathrm{mM}$ ) did not affect the measured mechanical properties of individual astrocytes in naive cultures $(49.2 \pm 4.4 \mathrm{kPa} ; n=18)$. Consistent with past observations, we observed a softening of the mechanical properties in stretch-injured, untreated astrocytes $(28.8 \pm 10 \mathrm{kPa} ; n=43)$. Pretreatment of cultures with PPADS restored the mechanical properties to naive, unstretched controls $(44.4 \pm 11.4 \mathrm{kPa}$ $[n=14 ;$ PPADS treated] vs. $49.2 \pm 4.4 \mathrm{kPa}[n=18$; treated naive]).

\section{Discussion}

In this study, we used AFM to investigate the stiffness of cultured astrocytes after mechanical injury. We first determined the mechanical threshold for astrocytes reactivity to appear $24 \mathrm{~h}$ following stretch injury, indicated by an increase in GFAP immunoreactivity. We found that this increase in GFAP immunoreactivity could be eliminated with apyrase, showing that ATP played a significant role in the mechanically induced reactivity of astrocytes. Non-nuclear areas of mechanically injured astrocytes showed a softening $24 \mathrm{~h}$ after injury; this softening response also appeared in astrocytes from the region of mechanical injury and the surrounding. Inhibiting P2 type purinergic receptors prevented the softening that appeared in the non-nuclear region of reactive astrocytes.

In general, our findings on the range and regional change in mechanical properties for individual astrocytes match most prior measurements for cultured astrocytes, and fit within the range of properties reported for other cell types. Although our measures of cell stiffness in the non-nuclear region of naive astrocytes were slightly higher than the 10-20-kPa range from a past measurement of astrocyte stiffness (Yamane et al., 2000), we used a different fitting approximation for our data that would yield slightly higher estimates of the mechanical properties compared to a more common conical approximation used in past AFM studies. Similarly, nuclear regions were proportionally softer in our samples to match prior observations (Radmacher et al., 1996; Ricci et al., 1997; Sato et al., 2000;
Yamane et al., 2000), even though the isolated nuclei are now known to be approximately an order of magnitude stiffer than the cytoplasm (Caille et al., 2002; Dahl et al., 2005). While the relative range of mechanical properties can change substantially when the viscoelastic properties are measured (Mathur et al., 2001; Lu et al., 2006), we chose only to measure the elastic properties of individual astrocytes in this study because of the known link between the elastic stiffness of a substrate and its role on neuronal outgrowth and differentiation (Flanagan et al., 2002; Engler et al., 2006). The influence of the complex modulus on similar neuronal outgrowth parameters remains unknown.

The role of ATP in producing reactive astrocytes is well established in past in vitro studies, and our data extend this past work by showing the central role that ATP-mediated signaling has in eliciting a reactive gliosis following mechanical stretch injury. Our unique observation that the reactivity can extend beyond the area of direct mechanical injury into the mechanical penumbra zone is consistent with injured astrocytes releasing ATP into the media and activating adjacent purinergic receptors. The role of mechanical injury in causing the release of ATP is well established from previous studies (Ahmed et al., 2000; Neary et al., 2003). The inhibition of reactive gliosis across the entire culture-including both the area of direct mechanical injury and the penumbra-by enzymatically degrading ATP in the extracellular media shows that ATP-mediated gliosis is a widespread mechanism for reactivity in our system. However, others have shown that glutamate release and subsequent activation of group I metabotropic glutamate receptors on astrocytes are important key steps in producing reactive gliosis in strain injured astrocytes, and that this pathway can occur even at modest levels of stretch (31\% peak) (Floyd et al., 2004). We cannot discount this possible mechanism of gliosis occurring at higher levels of stretch injury ( $>15 \%)$, especially considering that both glutamate and astrocytes can be release from cultured astrocytes (Parpura et al., 1994; Araque et al., 2000).

To our knowledge, this is the first data showing a new consequence of reactive astrocytes: the broad softening in a broad network of cells both within and distant from the site of mechanical injury. Given the extensive number of past studies using AFM on individual cells, there is some level of understanding of how structural changes in cytoskeletal elements lead to an adjustment in the bulk mechanical properties (Hoh and Schoenenberger, 1994; Rotsch et al., 1997; Costa and Yin, 1999; Sato et al., 2000; Collinsworth et al., 2002; Alonso and Goldmann, 2003). The most likely explanation for the observed softening of reactive astrocytes is the softness of intermediate filament networks, compared to other actin networks (Wagner et al., 2006) and the indirect role of GFAP on actin organization (Chang and Goldman, 2004). Microfilaments are the major load-bearing components of the cytoskeleton at small strains such as those measured here, with intermediate filaments only playing a secondary role (Trickey et al., 2004). Perturbing the actin cytoskeletal network with chemical treatments is a particularly powerful method to alter cell stiffness, leading to a drastic reduction in stiffness over time (e.g., 7× less stiff in $40 \mathrm{~min}$ ) (Rotsch and Radmacher, 2000). Intermediate filament reorganization is a much more subtle process which can induce actin re-organization (Chang and Goldman, 2004). Given the interplay between microfila- 
ment and intermediate filament dynamics, it is possible that our measured softening response in reactive astrocytes is simply a physical consequence of the extensive cytoskeletal reorganization that occurs within reactive astrocytes. In general, an increase in the GFAP immunoreactivity of astrocytes is a hallmark change associated with reactive astrocytes, but reactive astrocytes also show an increase in F-actin and $\gamma$-actinin, a common actin crosslinking protein, both in vivo and in vitro (Abd-El-Basset and Fedoroff, 1997; Moreels et al., 2007). Increased immunoreactivity may indicate a disaggregation of actin filaments, which would significantly reduce the measured elastic properties (Rotsch and Radmacher, 2000). Moreover, cellular stiffness is sensitive to the myosininduced tension generated in a cellular actin network (Martens and Radmacher, 2008), which may be an additional factor in reactive astrocytes that can explain the observed stiffness changes. Currently, though, the exact network structural mechanisms that underlie this softening response remain to be determined.

Perhaps most intriguing is considering the consequences of this softening response. In general, reactive astrocytes are considered important regulators of glial scar formation, with the compact network of glial cells physically blocking the regrowth of neurites through the scar (Pekny and Nilsson, 2005) and secreting, among other molecules, proteoglycans to limit regeneration (McKeon et al., 1999; Sandvig et al., 2004; Yiu and He, 2006). However, the major inhibitory aspect of the glial scar has been balanced with several reports more recently indicating the positive aspects of glial scar formation, including the role of GFAP in reactive astrocytes to provide neuroprotection in the ischemic penumbra (Li et al., 2008) and limiting excitotoxic damage (Hanbury et al., 2003; Otani et al., $2003,2006)$. The softening of reactive astrocytes lends one more potential beneficial aspect of glial reactivity, as cultured neurons prefer much softer substrates than typical cells (Balgude et al., 2001; Flanagan et al., 2002; Discher et al., 2005; Lu et al., 2006) and will show more neurite branching and extension on these softer surfaces. If this softening of reactive astrocytes is reflected in vivo after injury, these data suggest that reactive astrocytes may be a mechanically favorable substrate for neuronal regrowth and repair in the absence of other inhibitory factors. Indeed, GFAP knockout mice provide a more favorable environment for regeneration following spinal cord injury in vivo, and this effect becomes even more pronounced when a second common cytoskeletal protein found in astrocytes (vimentin) is also genetically deleted (Pekny et al., 1999; Pekny, 2001; Wilhelmsson et al., 2004; Pekny and Nilsson, 2005). Although these past studies did not definitively assess how these cytoskeletal alterations exactly affected the cellular or tissue level material properties, they nevertheless suggest that further exploring the role of matrix stiffness may lead to new insights into designing a permissive regenerative core. Considering and further manipulating these "durotactic" cues, in combination with controlling traditional chemotactic cues, may yield new insight onto a more optimal environment for regeneration processes that include sprouting, synaptogenesis, and axonal outgrowth.

\section{Acknowledgments}

Funds were provided by the National Institute of Health (grants RO1 HD41699 and NS35712).

\section{Author Disclosure statement}

The authors declare no competing financial interests.

\section{References}

Abbracchio, M.P., Ceruti, S., Bolego, C., Puglisi, L., Burnstock, G., and Cattabeni, F. (1996). Trophic roles of P2 purinoceptors in central nervous system astroglial cells. Ciba Found. Symp. 198, 142-148.

Abd-El-Basset, E., and Fedoroff, S. (1997). Upregulation of F-actin and alpha-actinin in reactive astrocytes. J. Neurosci. Res. 49, 608-616.

Ahmed, S.M., Rzigalinski, B.A., Willoughby, K.A., Sitterding, H.A., and Ellis, E.F. (2000). Stretch-induced injury alters mitochondrial membrane potential and cellular ATP in cultured astrocytes and neurons. J. Neurochem. 74, 1951-1960.

Alonso, J.L., and Goldmann, W.H. (2003). Feeling the forces: atomic force microscopy in cell biology. Life Sci. 72, 25532560.

Araque, A., Li, N., Doyle, R.T., and Haydon, P.G. (2000). SNARE protein-dependent glutamate release from astrocytes. J. Neurosci. 20, 666-673.

Araque, A., Parpura, V., Sanzgiri, R.P., and Haydon, P.G. (1998a). Glutamate-dependent astrocyte modulation of synaptic transmission between cultured hippocampal neurons. Eur. J. Neurosci. 10, 2129-2142.

Araque, A., Sanzgiri, R.P., Parpura, V., and Haydon, P.G. (1998b). Calcium elevation in astrocytes causes an NMDA receptor-dependent increase in the frequency of miniature synaptic currents in cultured hippocampal neurons. J. Neurosci. 18, 6822-6829.

Balgude, A.P., Yu, X., Szymanski, A., and Bellamkonda, R.V. (2001). Agarose gel stiffness determines rate of DRG neurite extension in 3D cultures. Biomaterials 22, 1077-1084.

Brambilla, R., Neary, J.T., Cattabeni, F., Cottini, L., D’Ippolito, G., Schiller, P.C., and Abbracchio, M.P. (2002). Induction of COX-2 and reactive gliosis by $\mathrm{P} 2 \mathrm{Y}$ receptors in rat cortical astrocytes is dependent on ERK1/2 but independent of calcium signalling. J. Neurochem. 83, 1285-1296.

Caille, N., Thoumine, O., Tardy, Y., and Meister, J.J. (2002). Contribution of the nucleus to the mechanical properties of endothelial cells. J. Biomech. 35, 177-187.

Chang, L., and Goldman, R.D. (2004). Intermediate filaments mediate cytoskeletal crosstalk. Nat. Rev. Mol. Cell. Biol. 5, 601-613.

Collinsworth, A.M., Zhang, S., Kraus, W.E., and Truskey, G.A. (2002). Apparent elastic modulus and hysteresis of skeletal muscle cells throughout differentiation. Am. J. Physiol. Cell. Physiol. 283, C1219-C1227.

Costa, K.D., and Yin, F.C. (1999). Analysis of indentation: implications for measuring mechanical properties with atomic force microscopy. J. Biomech. Eng. 121, 462-471.

Dahl, K.N., Engler, A.J., Pajerowski, J.D., and Discher, D.E. (2005). Power-law rheology of isolated nuclei with deformation mapping of nuclear substructures. Biophys. J. 89, 28552864.

Discher, D.E., Janmey, P., and Wang, Y.L. (2005). Tissue cells feel and respond to the stiffness of their substrate. Science 310, 1139-1143.

Engler, A.J., Sen, S., Sweeney, H.L., and Discher, D.E. (2006). Matrix elasticity directs stem cell lineage specification. Cell $126,677-689$.

Fiacco, T.A., and McCarthy, K.D. (2004). Intracellular astrocyte calcium waves in situ increase the frequency of spontaneous 
AMPA receptor currents in CA1 pyramidal neurons. J. Neurosci. 24, 722-732.

Flanagan, L.A., Ju, Y.E., Marg, B., Osterfield, M., and Janmey, P.A. (2002). Neurite branching on deformable substrates. Neuroreport 13, 2411-2415.

Floyd, C.L., Rzigalinski, B.A., Sitterding, H.A., Willoughby, K.A., and Ellis, E.F. (2004). Antagonism of group I metabotropic glutamate receptors and PLC attenuates increases in inositol trisphosphate and reduces reactive gliosis in straininjured astrocytes. J. Neurotrauma 21, 205-216.

Georges, P.C., Miller, W.J., Meaney, D.F., Sawyer, E.S., and Janmey, P.A. (2006). Matrices with compliance comparable to that of brain tissue select neuronal over glial growth in mixed cortical cultures. Biophys. J. 90, 3012-3018.

Halassa, M.M., Fellin, T., Takano, H., Dong, J.H., and Haydon, P.G. (2007). Synaptic islands defined by the territory of a single astrocyte. J. Neurosci. 27, 6473-6477.

Hanbury, R., Ling, Z.D., Wuu, J., and Kordower, J.H. (2003). GFAP knockout mice have increased levels of GDNF that protect striatal neurons from metabolic and excitotoxic insults. J. Comp. Neurol. 461, 307-316.

Hoh, J.H., and Schoenenberger, C.A. (1994). Surface morphology and mechanical properties of MDCK monolayers by atomic force microscopy. J. Cell Sci. 107, 1105-1114.

Kang, J., Jiang, L., Goldman, S.A., and Nedergaard, M. (1998). Astrocyte-mediated potentiation of inhibitory synaptic transmission. Nat. Neurosci. 1, 683-692.

Landau, L.D., and Lifshitz, E.M. (1970). Theory of Elasticity. Pergamon Press: Oxford, UK.

Lenz, G., Gottfried, C., Luo, Z., Avruch, J., Rodnight, R., Nie, W.J., Kang, Y., and Neary, J.T. (2000). P(2Y) purinoceptor subtypes recruit different mek activators in astrocytes. Br. J. Pharmacol. 129, 927-936.

Li, W.L., Yu, S.P., Ogle, M.E., Ding, X.S., and Wei, L. (2008). Enhanced neurogenesis and cell migration following focal ischemia and peripheral stimulation in mice. Dev. Neurobiol. 68, 1474-1486.

Lo, C.M., Wang, H.B., Dembo, M., and Wang, Y.L. (2000). Cell movement is guided by the rigidity of the substrate. Biophys. J. 79, 144-152.

Lu, Y.B., Franze, K., Seifert, G., Steinhauser, C., Kirchhoff, F., Wolburg, H., Guck, J., Janmey, P., Wei, E.Q., Kas, J., and Reichenbach, A. (2006). Viscoelastic properties of individual glial cells and neurons in the CNS. Proc. Natl. Acad. Sci. USA 103, 17759-17764.

Lusardi, T.A., Rangan, J., Sun, D., Smith, D.H., and Meaney, D.F. (2004). A device to study the initiation and propagation of calcium transients in cultured neurons after mechanical stretch. Ann. Biomed. Eng. 32, 1546-1558.

Martens, J.C., and Radmacher, M. (2008). Softening of the actin cytoskeleton by inhibition of myosin II. Pflugers Arch. 456, 95 100.

Mathur, A.B., Collinsworth, A.M., Reichert, W.M., Kraus, W.E., and Truskey, G.A. (2001). Endothelial, cardiac muscle and skeletal muscle exhibit different viscous and elastic properties as determined by atomic force microscopy. J. Biomech. 34, 1545-1553.

McKeon, R.J., Jurynec, M.J., and Buck, C.R. (1999). The chondroitin sulfate proteoglycans neurocan and phosphacan are expressed by reactive astrocytes in the chronic CNS glial scar. J. Neurosci. 19, 10778-10788.

Moreels, M., Vandenabeele, F., Dumont, D., Robben, J., and Lambrichts, I. (2007). Alpha-smooth mucle actin and nestin expression in reactive astrocytes in multiple sclerosis lesions: potential regulatory role of transforming growth factor-beta (TGF-beta1). Neuropathol. Appl. Neurobiol. 34, 532-546.

Munsch, N., Gavaret, J.M., and Pierre, M. (1998). Ca ${ }^{2+}$ dependent purinergic regulation of p42 and p44 MAP kinases in astroglial cultured cells. Biomed. Pharmacother. 52, 180186.

Neary, J.T., Kang, Y., Bu, Y., Yu, E., Akong, K., and Peters, C.M. (1999). Mitogenic signaling by ATP/P2Y purinergic receptors in astrocytes: involvement of a calcium-independent protein kinase $C$, extracellular signal-regulated protein kinase pathway distinct from the phosphatidylinositol-specific phospholipase C/calcium pathway. J. Neurosci. 19, 42114220.

Neary, J.T., Kang, Y., Tran, M., and Feld, J. (2005). Traumatic injury activates protein kinase b/akt in cultured astrocytes: role of extracellular ATP and p2 purinergic receptors. J. Neurotrauma 22, 491-500.

Neary, J.T., Kang, Y., Willoughby, K.A., and Ellis, E.F. (2003). Activation of extracellular signal-regulated kinase by stretchinduced injury in astrocytes involves extracellular ATP and P2 purinergic receptors. J. Neurosci. 23, 2348-2356.

Neary, J.T., McCarthy, M., Kang, Y., and Zuniga, S. (1998). Mitogenic signaling from P1 and P2 purinergic receptors to mitogen-activated protein kinase in human fetal astrocyte cultures. Neurosci. Lett. 242, 159-162.

Otani, N., Nawashiro, H., Fukui, S., Ooigawa, H., Ohsumi, A., Toyooka, T., Shima, K., Gomi, H., and Brenner, M. (2006). Enhanced hippocampal neurodegeneration after traumatic or kainate excitotoxicity in GFAP-null mice. J. Clin. Neurosci. 13, 934-938.

Otani, N., Nawashiro, H., Nomura, N., Fukui, S., Tsuzuki, N., Ishihara, S., and Shima, K. (2003). A role of glial fibrillary acidic protein in hippocampal degeneration after cerebral trauma or kainate-induced seizure. Acta Neurochir. Suppl. 86, 267-269.

Parpura, V., Basarsky, T.A., Liu, F., Jeftinija, K., Jeftinija, S., and Haydon, P.G. (1994). Glutamate-mediated astrocyte-neuron signalling. Nature 369, 744-747.

Pekny, M. (2001). Astrocytic intermediate filaments: lessons from GFAP and vimentin knock-out mice. Prog. Brain Res. 132, 2330.

Pekny, M., Johansson, C., Eliasson, C., Stakeberg, J., Wallen, A., Perlmann, T., Lendahl, U., Betsholtz, C., Berthold, C., and Frisen, J. (1999). Abnormal reaction to central nervous system injury in mice lacking glial fibrillary acidic protein and vimentin. J. Cell. Biol. 145, 503-514.

Pekny, M., and Nilsson, M. (2005). Astrocyte activation and reactive gliosis. Glia 50, 427-434.

Pelham, R.J., Jr., and Wang, Y. (1997). Cell locomotion and focal adhesions are regulated by substrate flexibility. Proc. Natl. Acad. Sci. USA 94, 13661-13665.

Powell, E.M., Meiners, S., DiProspero, N.A., and Geller, H.M. (1997). Mechanisms of astrocyte-directed neurite guidance. Cell Tissue Res. 290, 385-393.

Radmacher, M., Fritz, M., Kacher, C.M., Cleveland, J.P., and Hansma, P.K. (1996). Measuring the viscoelastic properties of human platelets with the atomic force microscope. Biophys. J. 70, 556-567.

Ricci, D., Tedesco, M., and Grattarola, M. (1997). Mechanical and morphological properties of living 3T6 cells probed via scanning force microscopy. Microsc. Res. Tech. 36, 165-171.

Rotsch, C., Braet, F., Wisse, E., and Radmacher, M. (1997). AFM imaging and elasticity measurements on living rat liver macrophages. Cell Biol. Int. 21, 685-696. 
Rotsch, C., and Radmacher, M. (2000). Drug-induced changes of cytoskeletal structure and mechanics in fibroblasts: an atomic force microscopy study. Biophys. J. 78, 520-55.

Sandvig, A., Berry, M., Barrett, L.B., Butt, A., and Logan, A. (2004). Myelin-, reactive glia-, and scar-derived CNS axon growth inhibitors: expression, receptor signaling, and correlation with axon regeneration. Glia 46, 225-251.

Sato, M., Nagayama, K., Kataoka, N., Sasaki, M., and Hane, K. (2000). Local mechanical properties measured by atomic force microscopy for cultured bovine endothelial cells exposed to shear stress. J. Biomech. 33, 127-135.

Sato, M., Theret, D.P., Wheeler, L.T., Ohshima, N., and Nerem, R.M. (1990). Application of the micropipette technique to the measurement of cultured porcine aortic endothelial cell viscoelastic properties. J. Biomech. Eng. 112, 263-268.

Shreiber, D.I., and Meaney, D.F. (1997). In vivo thresholds for mechanical injury to the blood-brain barrier. 41st Stapp Car Crash Conf. Proc.

Sneddon, I.N. (1965). The relation between load and penetration in the axisymmetric Boussinesq problem for a punch of arbitrary profile. Int. J. Eng. Sci. 3, 47-57.

Sofroniew, M.V. (2005). Reactive astrocytes in neural repair and protection. Neuroscientist 11, 400-407.

Trickey, W.R., Vail, T.P., and Guilak, F. (2004). The role of the cytoskeleton in the viscoelastic properties of human articular chondrocytes. J. Orthop. Res. 22, 131-139.

Ventura, R., and Harris, K.M. (1999). Three-dimensional relationships between hippocampal synapses and astrocytes. J. Neurosci. 19, 6897-6906.
Wagner, B., Tharmann, R., Haase, I., Fischer, M., and Bausch, A. (2006). Cytoskeletal polymer networks: the molecular structure of cross-linkers determines macroscopic properties. Proc. Natl. Acad. Sci. USA 103, 13974-13978.

Wang, H.B., Dembo, M., Hanks, S.K., and Wang, Y. (2001). Focal adhesion kinase is involved in mechanosensing during fibroblast migration. Proc. Natl. Acad. Sci. USA 98, 1129511300.

Wilhelmsson, U., Li, L., Pekna, M., Berthold, C.H., Blom, S., Eliasson, C., Renner, O., Bushong, E., Ellisman, M., Morgan, T.E., and Pekny, M. (2004). Absence of glial fibrillary acidic protein and vimentin prevents hypertrophy of astrocytic processes and improves post-traumatic regeneration. J. Neurosci. 24, 5016-5021.

Yamane, Y., Shiga, H., Haga, H., Kawabata, K., Abe, K., and Ito, E. (2000). Quantitative analyses of topography and elasticity of living and fixed astrocytes. J. Electron Microsc. (Tokyo) 49, 463-471.

Yiu, G., and He, Z. (2006). Glial inhibition of CNS axon regeneration. Nat. Rev. Neurosci. 7, 617-627.

Address reprint requests to:
David F. Meaney, Ph.D.
240 Skirkanich Hall
210 South $33^{\text {rd }}$ Street
Philadelphia, PA 19104-6321

E-mail: dmeaney@seas.upenn.edu 
